\title{
Towards Maximum Energy Efficiency of Carrier-Injection-Based Silicon Photonics
}

\author{
Clemens J. Krückel, Member, IEEE, Hanna Becker, Member, IEEE, Yoojin Ban, Member, IEEE, \\ Martijn J. R. Heck, Member, IEEE, Joris Van Campenhout, Member, IEEE, and \\ Dries Van Thourhout, Member, IEEE
}

\begin{abstract}
We present carrier-injection-based photonic switches, engineered for optical pulse distribution with maximum energy efficiency. We apply small-signal analysis and for the first time large-signal modelling to methodically optimize the switches for minimum energy consumption and to classify the electronic contributions from resistance, capacitance and diode. We present optimized electronic switch activation, which yields a sixfold reduction in energy consumption and we show how static power consumption becomes a negligible factor for optical pulse switching. We demonstrate that with adjusted phase shifter dimensions, MZI-based switches can operate with additional $50 \%$ enhanced energy efficiency with down to $4 \mathrm{pJ}$ per switching operation. We show even further efficiency improvement using ring-based designs, allowing an additional improvement of $50 \%$ in energy efficiency and we discuss the trade-off between efficiency and optical bandwidth associated to the Q-factor. We benchmark carrier-injection-based switches together with comparable technologies of the silicon photonics platform and identify carrier-injection to be the most suitable technology for pulse switching applications.
\end{abstract}

Index Terms-Electrooptic modulation, Equivalent circuits, Integrated optics, Optical switches, Phase modulation, Silicon on insulator technology

\section{INTRODUCTION}

A LL-optically addressable memory is an emerging digital memory technology that combines the advantages of random access memory (RAM) and flash memory and enables both ultra-fast and non-volatile data storage. Based on magnetoresitive RAM (MRAM), this memory technology stores information as the direction of magnetization in magnetic layers, which can be reversed to write binary information. Although this magnetization reversal can be done electrically, all-optical writing using short pulses appears far more energy efficient with a required writing energy potentially below $20 \mathrm{fJ}$ [1]. First demonstrations have been shown with single magnetic layers [2] as well as with layer stacks of magnetic tunnel junctions (MTJs) [3], which shows the potential of this memory technology for full integration.

With the scale-up to larger memory units, each memory

Manuscript submitted October 02, 2020.

C. J. Krückel (Clemens.Kruckel@UGent.be) and D. Van Thourhout (Dries.VanThourhout@UGent.be) are with the Photonics Research Group, Department of Information Technology, Ghent University - imec, Belgium. H. Becker (Hanna.Becker@eng.au.dk) and M. J. R. Heck (mheck@eng.au.dk) are with the Department of Engineering, Aarhus University, Denmark. Y. Ban (Yoojin.Ban@imec.be) and J. Van Campenhout (Joris.VanCampenhout@imec.be) are with IMEC, Leuven, Belgium.

This project has received funding from the European Union's Horizon 2020 research and innovation programme under grant agreement No 713481 . element has to remain optically addressable, which requires a suitably designed optical routing network. The network needs to be able to distribute short optical pulses (100 fs-10 ps) and additionally output sufficient pulse energy to write the MTJ elements. The latter imposes stringent limits on the insertion loss. Also, energy efficiency and speed are key parameters with a targeted repetition rate for the pulses in the order of $1 \mathrm{GHz}$.

For the integration of both MRAM cells and the optical routing network, a CMOS-compatible platform is the most suitable choice as it can provide electronic integration as well as photonic integration. Silicon photonics allows the fabrication of low-loss optical waveguides and electronically activated photonic switches in order to implement the routing network. While the optical bandwidth of these switches is mainly dependent upon design choices, parameters like insertion loss, speed and device dimensions are primarily given by the technology of its fundamental building block: the optical phase shifter. For the implementation of phase shifters in silicon, three approaches, all based on the plasma-dispersion effect, are available: carrier-depletion [4], [5], carrier-accumulation [6] and carrier-injection [7]. Based on performance data reported in the literature, carrier-injection technology appears to be the most suitable contestant for the given demands. With this technology a $\pi$-phase shift can be achieved with $<1 \mathrm{~mm}$ devices, drive voltages around $1 \mathrm{~V}$ and insertion loss of 0 $1 \mathrm{~dB}$ [8], thus offering compact, efficient and low-loss phase shifters.

Although this technology has been studied thoroughly for alternating current (AC) and direct current (DC) operations, the performance evaluation of the carrier-injection technology for applications that focus on the routing of low-duty cycle pulse trains is still missing. In this paper, an extension of our work in [9] and [10], we present our findings that are geared towards the energy efficient switching of optical pulses used for all-optically addressable memory. However, the provided study of carrier-injection-based silicon photonics is universal and applicable to other applications like wavelength-division multiplexing (WDM) or data modulation.

Additionally, up until now predictions of the energy consumption in this technology considered only one of the following three contributions: Joule heating in the load resistance [11], [12], simplified calculation of the device capacitance [13]-[15] or estimation of the total injected charge [16]. We introduce a more complete study of the energy consumption which is based on small-signal analysis and large signal modelling 
that takes into account the complete device capacitance, Joule heating and moreover accounts for charge losses during the device activation.

Furthermore, although the limited modulation speed of this technology has been addressed with pre-emphasis driving schemes [17], their impact on the energy consumption has not been evaluated. We include this insight in our evaluation and discuss the impact of switch design, driving scheme and static power consumption on the energy efficiency of phase shifters and switches.

\section{Electrostatics IN PIN-DIODE-BASED PHASE SHIFTERS}

As introduced above, optical phase shifters in silicon can be realized by leveraging the plasma-dispersion effect [18]. This effect relates a variation in the carrier density to a change in refractive index $n$ and optical attenuation $\alpha$. Based on empirical studies, these relations for bulk silicon and for a wavelength of $1550 \mathrm{~nm}$ are found to be [19]:

$$
\begin{gathered}
-\Delta n=5.4 \times 10^{-22} \Delta N_{e}^{1.011}+1.53 \times 10^{-18} \Delta N_{h}^{0.838} \\
\Delta \alpha=8.88 \times 10^{-22} \Delta N_{e}^{1.167}+5.84 \times 10^{-20} \Delta N_{h}^{1.109}
\end{gathered}
$$

In the equations $\Delta N_{e}$ and $\Delta N_{h}$ account for electron and hole densities respectively $\left(1 / \mathrm{cm}^{3}\right)$. The induced optical attenuation $\alpha$ is defined per unit length $(1 / \mathrm{cm})$.

The carrier-injection-based phase shifters presented in this work are designed with a pin-diode embedded into a single mode rib waveguide as illustrated in Fig. 1.a. The devices are fabricated within the iSiPP50G platform of imec [20] and are based on rib waveguides with a center dimension of $220 \times 450 \mathrm{~nm}^{2}$ and a slab height of $70 \mathrm{~nm}$. Under forward bias, minority charge carriers diffuse from the doped slab areas into the waveguide region, where the optical mode is confined. This induced change in carrier concentration causes an index change according to Eq. 1 and thus introduces a phase shift of

$$
\Theta=\Gamma \cdot k \cdot \Delta n \cdot L .
$$

with the wavenumber $k$, the device length $L$, and $\Gamma$ accounting for the overlap of the mode profile with the carrier distribution [21], which is supposed to be homogeneous over the waveguide cross-section. For a better characterization of the phase shifter on device level, it is convenient to relate the total phase shift and absorption loss to the overall electric charge present in the whole structure. The total charge $Q$ that is present in the waveguide can be related to the charge carrier density using

$$
Q=q_{e} \cdot \Delta N \cdot A \cdot L
$$

with elementary charge $q_{e}$ and waveguide cross-section area $A$. The carrier concentration of electrons and holes is accounted for via $\Delta N$. Assuming a waveguide cross-section of $220 \times 450 \mathrm{~nm}^{2}$, Fig. 1.b and c show the total phase shift and associated loss as a function of charge for phase shifter lengths varying from 250 to $1400 \mu \mathrm{m}$. In the following, the negative phase shift associated with carrier-injection is presented as an absolute value. The length dependency arises from the nonlinearity in the empirical relations given by Eq. 1 and 2. In essence, for the same total charge, a longer diode displays a

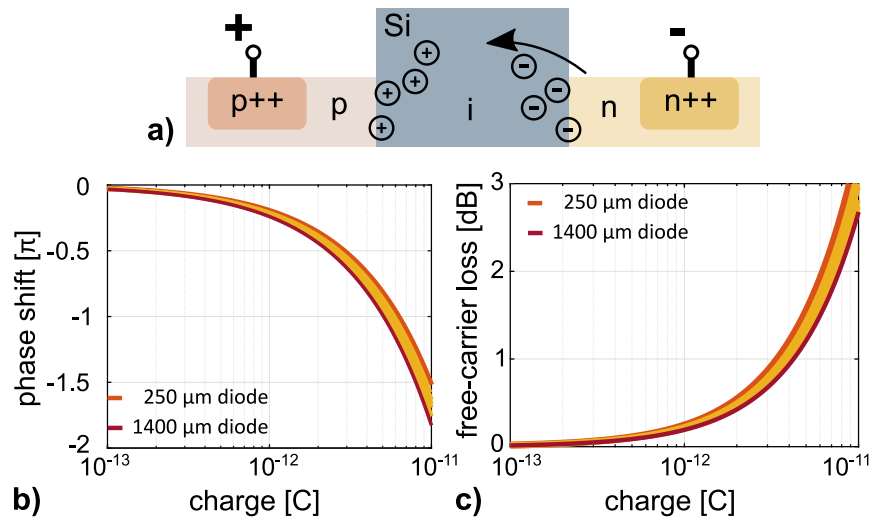

Fig. 1: a) Illustration of the cross-section of a carrier-injectionbased phase shifter operated under forward bias. b) and c) Theoretical phase shift and free-carrier loss in silicon waveguides $\left(220 \times 450 \mathrm{~nm}^{2}\right)$ vs. injected electric charge. Plotted for device diode lengths from 250 to $1400 \mu \mathrm{m}$

slightly higher optical phase shift at lower absorption, which results in fundamentally reduced electronic energy consumption but less compact devices.

For the characterization of a phase shifter on device level it is important to correlate the amount of injected charge to the applied forward voltage and the resulting diode current. Details about the charge-voltage relation can be obtained through the diode capacitance that is extracted from smallsignal measurements. In these measurements, the small-signal admittance $Y$ is determined, which contains information about the capacitive as well as the resistive properties of the diode. Eq. 5 describes how these properties are connected and Fig. 2.a shows the associated small-signal equivalent circuit model [22].

$$
Y=1 / R_{s}+G_{D}+j \omega\left(C_{J}+C_{D}\right)=i / u_{a}
$$

The equation includes series resistance $R_{s}$, diode conductivity $G_{D}$, junction capacitance $C_{J}$ and diffusion capacitance $C_{D}$. The admittance represents the ratio of small-signal current $i$ and small-signal voltage $u_{a}$, which are both alternating current (AC) signals. The small-signal current originates from charge oscillations in response to the alternating electrical field caused by $u_{a}$. These charge oscillations arise from both majority carriers in the depletion regions and minority carriers that are injected towards the waveguide center (see Fig. 1.a). These effects are captured by the junction and diffusion capacitance respectively. For the small-signal measurements, the AC signal amplitude was set to $35 \mathrm{mV}_{\mathrm{pp}}$ and data are presented here for a small-signal frequency of $100 \mathrm{kHz}$. By superimposing a DC bias on the small-signal voltage, it is possible to measure the voltage-dependent characteristics of $G_{D}, C_{J}$ and $C_{D}$. The DC bias is scanned in the relevant voltage range from 0 to $1 \mathrm{~V}$. We measured the device characteristics for various diode lengths from 250 to $1400 \mu \mathrm{m}$ and are exemplifying these results on the basis of the results for a $1000 \mu \mathrm{m}$ long diode in the following. The total measured capacitance, together with fitted $C_{J}$ and $C_{D}$, versus applied bias voltage is shown in Fig. 2.b. At low bias voltage levels, the junction capacitance 


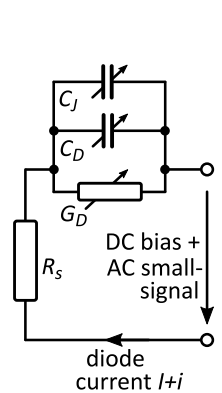

a)

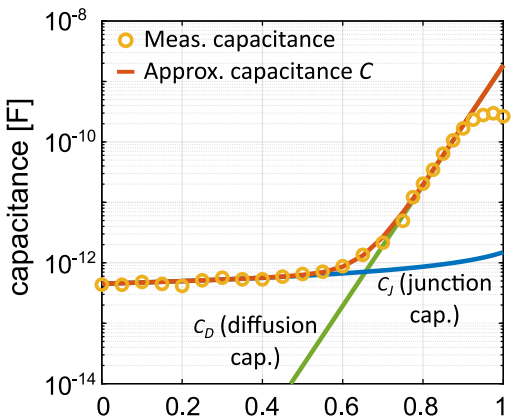

b)

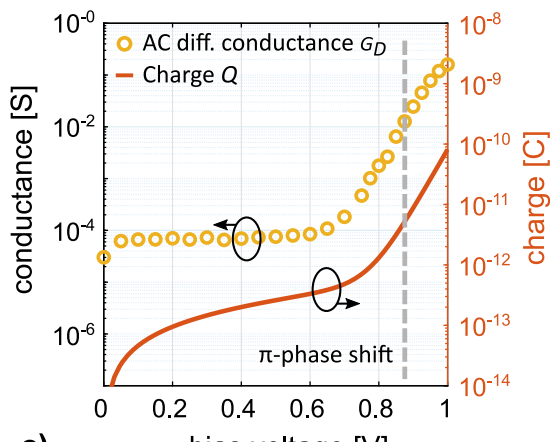

c)

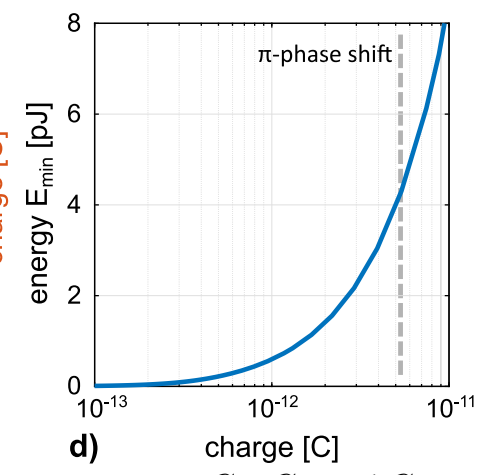

Fig. 2: a) Small-signal equivalent circuit for the pin-diode phase shifter. The voltage-dependent components $C_{J}, C_{D}$ and $G_{D}$ are respectively the junction capacitance, the diffusion capacitance and the diffusion conductance. $R_{s}$ is the series resistance. The diode current is a superposition of DC current $I$ and small-signal current $i$. b) Measured and approximated device capacitance as a function of applied DC bias voltage $(1000 \mu \mathrm{m}$ diode length). c) Approximated small-signal diffusion conductance and calculated diode charge vs. applied DC bias voltage $(1000 \mu \mathrm{m}$ diode length). The dotted line indicates $\pi$-phase shift operation at around $0.87 \mathrm{~V}$. d) Stored capacitive energy in relation to its stored diode charge. The dotted line indicates $\pi$-phase shift operation at around $5.3 \cdot 10^{-12} \mathrm{C}$.

dominates and its voltage dependency is approximated by $C_{J}=C_{J 0} /\left(1-U_{a} / U_{b i}\right)$ [22], with $C_{J 0}$ being the capacitance at $0 \mathrm{~V}, U_{a}$ the applied DC bias voltage and $U_{b i}$ the builtin voltage $(\sim 1.1 \mathrm{~V})$. At around $0.6 \mathrm{~V}$, the contribution from the diffusion capacitance becomes relevant. Throughout this work the diffusion capacitance will be approximated with $C_{D}=10^{p_{0}}+p_{1}$, where $p_{0}$ and $p_{1}$ represent slope and offset of the capacitance in the voltage range between 0.7 and $0.9 \mathrm{~V}$. The total approximated capacitance $C$, the sum of $C_{J}$ and $C_{D}$, is a good fit to the measured capacitance, as shown in Fig. 2.b. The saturation of the measured capacitance at higher voltages is assumed to be an artefact of the measurements where the high diffusion conductance makes the capacitance evaluation difficult, and is not taken into further consideration. The voltage-dependent AC diffusion conductance $\left(G_{D}\right)$ is shown in Fig. 2.c. It is a good approximation of the IV-relation in DC operation (Section IV) above $0.7 \mathrm{~V}$. At lower voltage levels the current flow as obtained from the small-signal analysis is overestimated. This is due to majority carrier oscillations, which give no contributions to DC current under steady-state operations.

Based on the approximated capacitance extracted from the small-signal measurements, the charge present in the device for a given voltage is calculated as

$$
Q=\int_{0}^{U_{a}} C_{J}(u)+C_{D}(u) d u .
$$

This calculated charge is shown in Fig. 2.c for the measured bias voltage range. How the evaluated charge in the device relates to the optical phase shift and attenuation can then be found from Fig. 1.b and c [16], [17]. As seen in these figures, the phase shift target sets the amount of charge that has to be moved into the waveguide area. For example, in the $1000 \mu \mathrm{m}$ diode a $\pi$-phase shift is predicted for an injected charge of $5.3 \cdot 10^{-12} \mathrm{C}$. Equally, this requires a forward-bias voltage of around $0.87 \mathrm{~V}$ as indicated in Fig. 2.c.

Based on the measured capacitance-voltage relation, it is now possible to estimate the energy that has to be delivered to reach a dedicated charge level in the diode:

$$
E_{\text {min }}=\int_{0}^{U_{a}}\left(C_{J}(u)+C_{D}(u)\right) u d u .
$$

For devices with a voltage-independent capacitance, e.g. for phase-shifters based on carrier-depletion [28], this relation can be simplified to $E_{\min }=0.5 C \cdot V^{2}$. However, for carrierinjection devices, with their voltage-dependent capacitance, the full integral has to be evaluated. The result, for a $1000 \mu \mathrm{m}$ long diode, is shown in Fig. 2.d, which plots the energy $E_{\text {min }}$ vs. charge and shows that for obtaining a $\pi$-phase shift, a minimum energy of around $4 \mathrm{pJ}$ is required. This value is fundamentally limited by the efficiency of the plasmadispersion effect as given by Eq. 1 .

In classical terms, this value corresponds to the energy that is stored in a capacitor. Here, we consider this as the energy required to reach a given phase shift and to activate the switch, which we define as the dynamic energy consumption of the device [23]. It is important to realize, that $E_{\min }$ is the bottom limit of the dynamic energy consumption. Additional contributions to this energy arise from series resistance and diode conductance. Their magnitude will be discussed in Section IV, where we additionally introduce the required energy to keep the switch activated which is related to the static power consumption of the device.

\section{DESIGN-DEPENDENT PHASE SHIFT AND ENERGY CONSUMPTION DURING SWITCHING OPERATION}

In order to realize photonic switches, phase shifters are commonly embedded in architectures like the Mach-Zehnder interferometer (MZI) [8], [24] or ring-resonators [25]. While the electrical bandwidth of these switches is largely determined by carrier dynamics of the phase shifter technology, properties like optical bandwidth and the required phase shift for switching are primarily impacted by the switch design. 


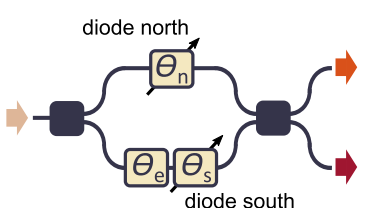

output 1 a)

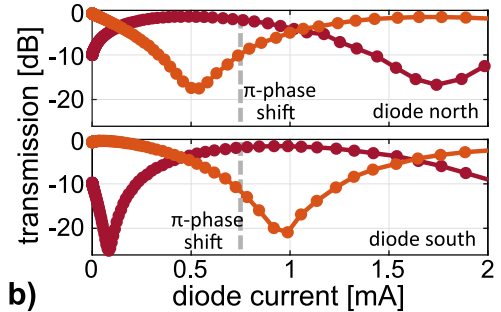

Fig. 3: a) Illustration of a MZI-based 1x2 switch. The switch is designed with tunable phase shifters in both Mach-Zehnder arms $\left(\theta_{\mathrm{n}}\right.$ and $\left.\theta_{\mathrm{s}}\right)$ and an extra phase delay in the southern $\operatorname{arm}\left(\theta_{\mathrm{e}}\right)$. b) Transmission of the MZI-switch $(500 \mu \mathrm{m}$ diode length) at both output ports in dependence of the applied diode current. Upper graph - modulation of diode north, lower graph - modulation of diode south [as defined in a)].

Both architectures (MZI- and ring-based) exhibit their respective advantages and disadvantages and in the following we will show how design choices affect switching voltage and current and as a result the energy consumption.

MZI-based switches show a sinusoidal response in relation to the phase difference between both arms [24] and offer a large optical bandwidth of several 10s of nm [8], suitable to switch short optical pulses with durations in the sub-100 fs regime. For a 1x2 MZI as shown in Fig. 3.a, ideally the maximum extinction ratio (ER) is obtained for zero-injected diode current. However, in practical devices, small fabrication variations might induce a small shift in the measured response, as is visible in the experimental results depicted in Fig. 3.b. The presented device contains pin-phase shifters with a length of $500 \mu \mathrm{m}$ and additionally a static phase delay of $0.2 \pi$ between both arms. In some cases, this additional static phase delay can be used to our advantage. E.g. in this case, when driving the north diode and exploiting the broad maximum around zero injection, a smaller current is required to obtain full switching than would be needed in a perfect device. The deviation from the balanced MZI-switch is visualized in the figure (Fig. 3.b). In general, the on-state of these switches is achieved around the $\pi$-phase shift which requires $0.75 \mathrm{~mA}$ diode current at an applied voltage of $0.92 \mathrm{~V}$. The ER, the power difference between both output ports, reaches a maximum of around $20 \mathrm{~dB}$. Minimum insertion loss of the deactivated (off-state) and activated (on-state) device are $\sim 0.3 \mathrm{~dB}$ and $\sim 1.4 \mathrm{~dB}$.

Next, we evaluate how the condition for switching changes when modifying the phase shifter length. We take the $\pi$-phase shift requirements of a balanced MZI-switch as a benchmark. Based on the model established in Section II, we calculate the amount of electric charge required in the diode to achieve the phase shift target of $\pi$, see Fig.4.a. The plot indicates lower charge requirements for longer diodes originating from the nonlinear relation of carrier-density and refractive index change favoring longer diodes (see figure inset). This reduction in injected charge translates directly into lower voltage and current requirements in the diode (Fig. 4.b). Consequently, longer diodes display a reduction in static power consumption when actuated. The data in this graph was extracted from current-dependent loss measurements of stand-alone phase
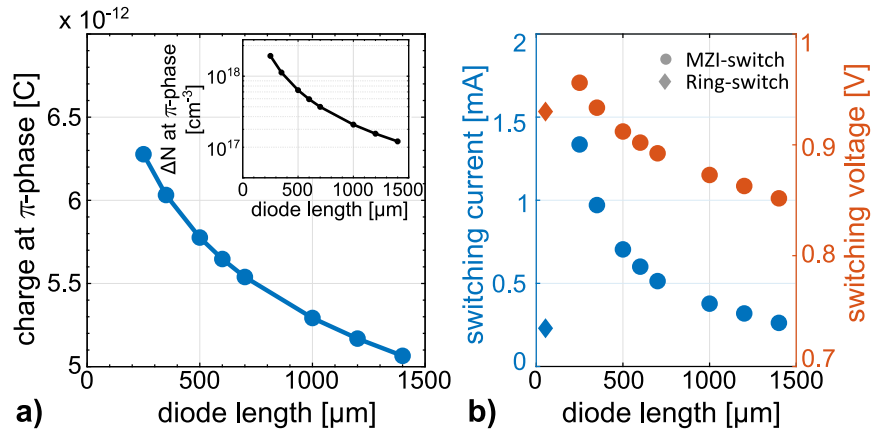

Fig. 4: a) Calculation of the electric charge requirements for a $\pi$-phase shift as function of phase shifter diode length. The inset shows the corresponding carrier concentration $\Delta N$ in the phase shifter diode. b) Experimentally evaluated current and voltage requirements for switching as a function of phase shifter length, assuming a full $\pi$-phase shift for the MZI-switch and $0.1 \pi$-phase shift for the ring-switch respectively.

shifters with varying lengths, relating absorption to phase shift using Eq. 1 and 2. The results obtained for the $500 \mu \mathrm{m}$ long phase shifter agrees well with the experimental curves of the MZI-switch presented in Fig. 3.b.

The ring-switch architecture, shown in Fig. 5.a, includes a tunable phase shifter (pin-diode) within the waveguide section of the ring-resonator. In the experimental examination of this design, we use a ring with a radius of $15 \mu \mathrm{m}$, a gap between ring and bus waveguide of $240 \mathrm{~nm}$ and a phase shifter length of $\sim 55 \mu \mathrm{m}$. Optical transmission at both output ports depends on the location of the resonator resonance with respect to the wavelength of the inserted probe, and is adjusted via the pin-diode. Fig. 5.b shows the transmission for both output ports, for 3 different applied diode currents $(0,0.5$ and $2 \mathrm{~mA}$ ). Switching operation of the device is demonstrated experimentally by switching a 5 ps-long laser pulse centered at $1555.3 \mathrm{~nm}$ wavelength. The throughput at both ports as a function of the phase shifter current is shown in Fig. 5.c. As the switch is operated on-resonance, maximum throughput in the off-state is achieved at output 1 (drop-port). This operation scheme has the advantage of avoiding free-carrier absorption

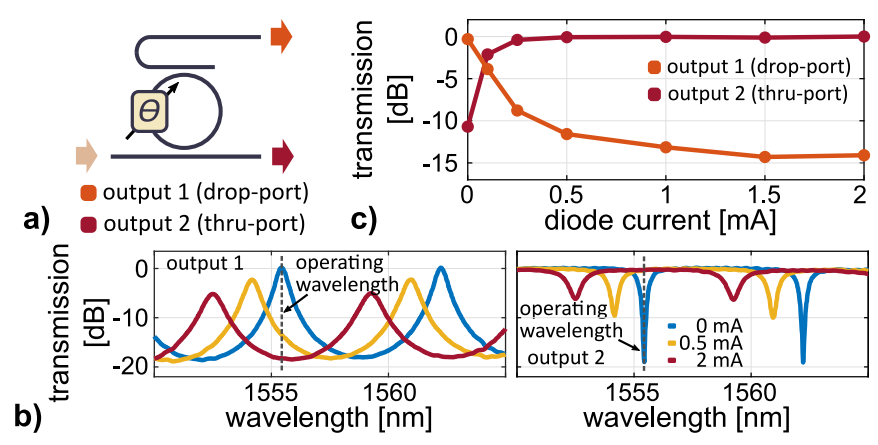

Fig. 5: a) Sketch of the ring-based 1x2 switch. b) Wavelengthdependent transmission of the ring-switch outputs at three different phase shifter currents $(0,0.5$ and $2 \mathrm{~mA})$. c) Transmission of the ring-switch at both output ports vs. the applied diode current. Optical probe signal at $1555.3 \mathrm{~nm}$ wavelength. 

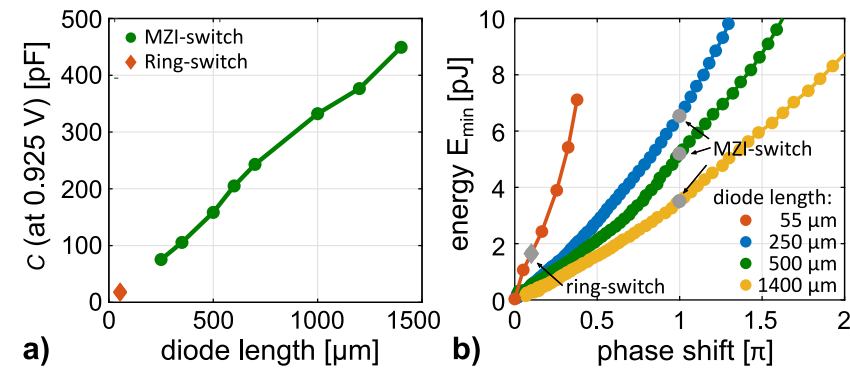

Fig. 6: a) Diode capacitance $(C)$ vs. phase shifter length evaluated at a bias voltage of $0.925 \mathrm{~V}$. b) Energy stored in the diode capacitance as a function of generated optical phase shift for four distinct diode lengths.

loss during the switching process, resulting in higher overall output power levels at both ports. For the ring, we define phase shifter activation (on-state) at an extinction ratio of $10 \mathrm{~dB}$ similar to the off-state, which is sufficient for our application. This switch-state requires a phase shift of $0.1 \pi$ with $0.23 \mathrm{~mA}$ diode current and $0.93 \mathrm{~V}$ bias voltage. The on-state requirements for phase shift and diode current are significantly lower compared to the MZI-switch. This arises from the resonant nature of the ring-switch. Here, we use a device with a quality factor $\mathrm{Q} \sim 3000$, which results in comparably lower static power consumption, see Fig. 4.b, and a more compact device in comparison to the MZI-switches. The drawback is a limited optical bandwidth of $\sim 0.5 \mathrm{~nm}$. This restricts the switching of pulses shorter than $5 \mathrm{ps}$ and increases the minimum insertion loss for output 1 and 2 to $0.3 \mathrm{~dB}$ and $0.4 \mathrm{~dB}$. This insertion loss can be optimized by tuning the Qfactor and in Section $\mathrm{V}$ we further discuss the Q-factor impact on the performance when switching short pulses.

In order to assess the energy efficiency of the two switch designs, we calculate the energy consumption in dependence on the phase shift requirements and the phase shifter length. For this, we use Eq. 3-4 as well as Eq. 6-7 to link diode charge, phase shift and energy consumption. The evaluation includes the measured small-signal capacitance, which identifies a linear relation of diffusion capacitance vs. diode length as shown in Fig. 6.a. Although the figures indicates an increasing capacitance with diode length, longer phase shifters are clearly more energy efficient as shown in Fig. 6.b. As an example, to reach a $\pi$-phase shift, an almost $50 \%$ reduction in energy consumption is obtained when increasing the diode length from 250 to $1400 \mu \mathrm{m}$. For the ring-switch, despite using a shorter diode that is far less efficient, the energy cost is considerably lower as only a $0.1 \pi$-phase shift is required for switching. This illustrates the benefits of reduced phase shift requirements for switching, which can also be achieved by using a slightly unbalanced MZI-structure as mentioned above.

\section{DETERMINATION OF ENERGY CONSUMPTION BASED ON LARGE-SIGNAL MODEL}

Thus far only the energy required to charge the voltagedependent capacitance was taken into account to approximate the dynamic energy consumption. This calculation assumed an instantaneous injection of charge into the diode without considering the transient response to reach the on-state. During the activation of the switch additional charge streams through the diode causing an additional contribution to the energy consumption. As we will see, this energy contribution strongly depends on the applied electrical driving scheme. For our application it is the best to operate the carrier-injection-based switches with large-signal modulation using a return-to-zero approach. This has two advantages: first, it eliminates power consumption in the off-state and second, it allows for the minimization of dynamic energy consumption to activate the switch and retain its on-state. This is especially useful in case of switching optical signal pulse trains.

In order to optimize the driving scheme, we implement a large-signal model. Besides activation time, the model reveals how much energy is consumed by the different electrical components of the phase shifter and allows to compare these with the previously studied capacitive energy consumption. The used large-signal equivalent circuit model is shown in Fig. 7.a [22], [26]. It contains three electrical components: the series resistance $R_{s}$, the voltage-dependent capacitance $C$ and an ideal diode. It is convenient to separate series resistance and the ideal diode in the model and retrieve the current-voltage (IV) relation of the real diode via

$$
I=I_{0} e^{\left(U_{a}-I \cdot R_{s}\right) q_{e} / n_{i d L} \cdot k_{b} \cdot T} .
$$

with applied voltage $U_{a}$, diode ideality factor $n_{i d L}$, saturation current $I_{0}$, and thermal voltage $k_{b} \cdot T / q_{e}$. The diode parameters $\left(R_{s}, n, I_{0}\right)$ are captured from the diodes measured IV characteristics (Fig. 7.b) according to [22], here shown for a $1000 \mu \mathrm{m}$ diode. The series resistance was measured to be around 5-10 $\Omega$ for diodes with a length from $1400 \mu \mathrm{m}$ down to $250 \mu \mathrm{m}$, respectively. Its impact on the real diode current can be seen in Fig. 7.b as a deviation from the linear slope at voltage levels beyond the built-in voltage of the diode. The capacitance $C$ changes with the voltage drop across the ideal diode. It represents the charge present in the diode and impacts the turn-on and turn-off transient behaviour. The circuit model is implemented in Simulink [27], where timeresolved simulations are carried out. The response of the diode to a time-dependent voltage sequence is simulated with a sequence of two and three voltage levels i.e. a square waveform and a pre-emphasized square waveform respectively [17].

The large-signal model is experimentally verified by measuring the optical attenuation of stand-alone phase shifters. The direct link between changes in real and imaginary part of the refractive index (see Eq. 1 and 2) allows then to derive the phase shift response. The results for a $1000 \mu \mathrm{m}$ long diode are presented here. As part of the model, we use the measured relation between diode current and optical insertion loss in the phase shifter. An absorption loss target of $2 \mathrm{~dB}$ was chosen, close to the expected loss at $\pi$-phase shift. We chose five different pre-emphasis voltage levels between 0.9 and $2 \mathrm{~V}$ as shown in Fig. 7.c (top). The duration of the preemphasis corresponds to the time it takes to reach the loss target. Succeeding the pre-emphasis is a $0.9 \mathrm{~V}$ level for a duration of $1 \mathrm{~ns}$ corresponding to the on-state with a fixed 
transmission loss of $2 \mathrm{~dB}$. The experimental electric driving signal is generated with an Arbitrary Waveform Generator (AWG) with 0.2 ps rise- and fall-time and a $50 \Omega$ internal load. The optical signals after the photonic device were captured with a photodetector $(30 \mathrm{GHz})$ and a high-speed sampling oscilloscope. Measured and simulated transmission transients are displayed in Fig. 7.c (bottom), indicating that speed-limitations mainly arise from the turn-on transient rather than turn-off transient. The figure shows the expected faster response of the device when activated with a larger pre-emphasis voltage. This trend is summarized in Fig. 7.d, which indicates that already a small increase in pre-emphasis voltage towards around $1 \mathrm{~V}$ results in a large reduction in turn-on time. The figure also displays the increased current charging the capacitance $I_{C}$ (Fig. 7.a), causing a faster charge injection into the diode and thus the shorter response time [17], [22].

We utilize this experimentally verified large-signal model to predict the energy consumption in each of the electronic components (i.e. series resistance, ideal diode and capacitance) as follows. The simulation model provides the time-dependent power consumption as calculated from the temporal voltage drop and current flow. Evaluating the integral of power over time-to-on-state yields the energy consumption for activation. We can then relate the energy consumption in each component to a specific driving scheme, in this case to the chosen preemphasis driving voltage $V_{\text {pre }}$. This is illustrated for the energy required to reach a $\pi$-phase shift as it leads to switching with maximum extinction ratio in MZI-switches. As an on-state target, we use the diode current from Fig. 4.b in the simulation model. The individual contributions of resistance, capacitance and the ideal diode are shown in Fig. 7.e. The plot reveals that the capacitive energy consumption is independent of the driving scheme. As expected, the value matches the predicted energy from Section II. However, the energy that is consumed by the ideal diode of the device is significantly lower at larger pre-emphasis voltages. This is explained by the much shorter activation time, which significantly reduces the current leaking through the device before reaching the on-state. At higher $V_{\text {pre }}$, this benefit is countered with an increase in Joule heating originating from the higher driving current, reaching $0.2 \mathrm{pJ}$ for a pre-emphasis voltage of $2 \mathrm{~V}$. The Joule heating for the presented driving schemes is significantly lower than other values reported in literature [11], benefiting from the low series resistance of the device. This contribution is much smaller than the capacitive energy and comparable to the static energy consumption i.e. the energy required to maintain the onstate. Holding the switch activated for $1 \mathrm{~ns}$ consumes around $0.4 \mathrm{pJ}$. Thus, although carrier-injection-based phase shifters display static power consumption when being operated, this becomes nonrelevant when switching short pulses because of the negligible on-state duration that is required. From Fig. 7.e, it becomes clear that for the most energy efficient driving scheme ( $V_{\text {pre }} \sim 1.4 \mathrm{~V}$ ), the total energy consumption is limited by the capacitive part. For this driving scheme the turn-on time is $0.7 \mathrm{~ns}$, enabling switching with $\mathrm{GHz}$ speed.

The total energy consumption obtained from the large-signal model is displayed in Fig. 7.f, summarizing the contributions from all three electrical components. The figure displays a selection of the experimentally characterized switches including MZI-switches with different diode lengths as well as the ringbased switch. It can be seen that for all devices the energy consumption approaches the capacitive energy consumption (see Section II), when being operated with an optimized electrical activation scheme. For the most efficient driving scheme, we evaluated a reduction in energy consumption by a factor of six compared to a standard square-waveform activation. More details about this are provided in the Appendix. Additionally, the figure indicates the lower energy consumption of longer, more efficient, phase shifters, as discussed above. The minimum total energy consumption for all evaluated devices is summarized in Fig. 7.g. The figures shows a $50 \%$ reduction in energy consumption when moving to longer phase shifters and an additional $50 \%$ improved efficiency when utilizing the ring-switch design. It is worth mentioning, that the minimum
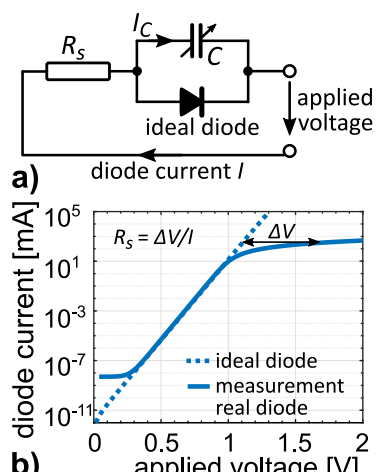

b) applied voltage [V]
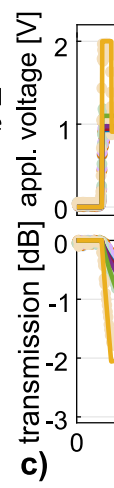

- measurements - -simulations
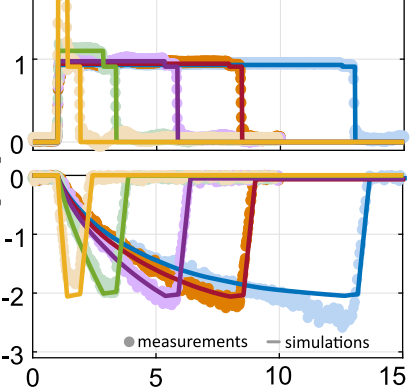

time [ns]

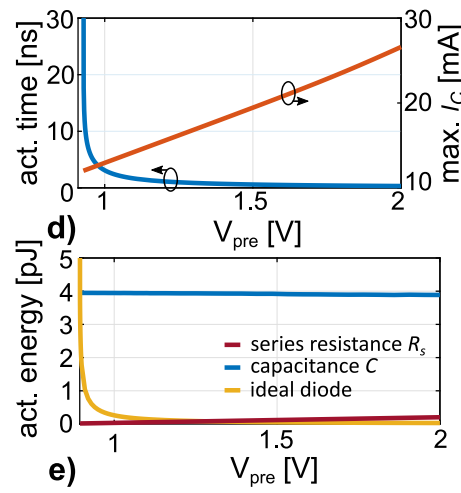

e)

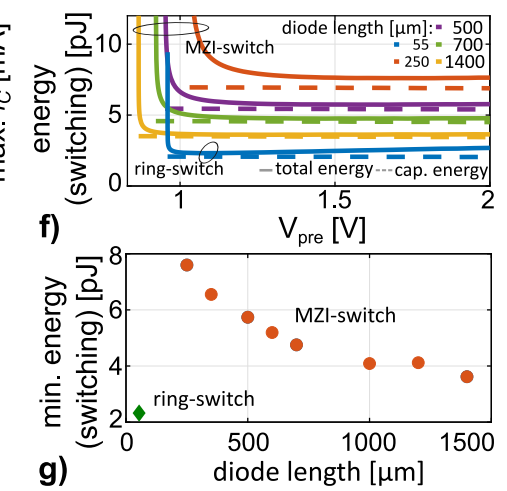

Fig. 7: a) Large-signal equivalent circuit model of pin-diode. b) Measured current voltage relation of $1000 \mu \mathrm{m}$ phase shifter diode and extracted ideal diode characteristics. c) Simulation and measurement of electronic driving signal and optical transmission of a $1000 \mu \mathrm{m}$ stand-alone phase shifter for reaching a loss of $2 \mathrm{~dB}$. d) Simulated activation time (loss target $2 \mathrm{~dB}$ ) and the corresponding maximum capacitance current $I_{C}$ as a function of pre-emphasis voltage level ( $V_{\text {pre }}$ ). e) Contributions to the activation energy ( $\pi$-phase shift target) as function of the pre-emphasis voltage level. f) Total energy consumption to reach switching versus strength of the pre-emphasis. g) Minimum energy consumption as a function of phase shifter length. 
activation time of $0.7 \mathrm{~ns}$ is similar for all designs [10].

\section{EXPERIMENTAL DEMONSTRATION OF PULSE-SWITCHING OPERATIONS}

In the following, we show the potential of the carrierinjection technology for switching applications with a pulse switching demonstration. Spatial pulse distribution is shown in the two-port architectures based on Mach-Zehnder interferometers and ring-resonators. The large optical bandwidth of MZI-based switches gives the possibility to switch ultrashort pulses. In the experiment, we use a pulsed laser source centered around $1550 \mathrm{~nm}$ with a pulse-width around $80 \mathrm{fs}$ and a repetition-rate of $20 \mathrm{MHz}$. The optical phase shifter (500 $\mu \mathrm{m}$ length) in one arm of the MZI-switch is driven by the AWG as mentioned above. For fast switch-activation, a pre-emphasis driving scheme was used and then combined with an on-state level duration of $0.5 \mathrm{~ns}$. Fig. 8.a shows the experimental results when activating the switch with a $2 \mathrm{~V}$ preemphasis voltage (maximum of the AWG). The experiment was set up to route every second pulse to the other port as shown by the two consecutive pulses displayed in the figure. It is worth mentioning, that for this demonstration low pulse intensities were used to avoid nonlinear effects in the waveguide. For a better recording of the transient response during the switch process, we launched $\mathrm{CW}$-light into the system in addition to the laser pulses. Moreover, this practice allows for a straightforward alignment of timing between optical pulse train and electrical activation sequence in the time domain. The analysis of the transient response was carried out for various driving schemes which are summarized in Fig. 8.b. The figure identifies the expected turn-on behavior, which is in good agreement with the simulations of the large-signal model. The slight mismatch at lower voltages could be explained with a variation in voltage drop across the diode originating, for instance, from a varying probe contact. The turn-on time of

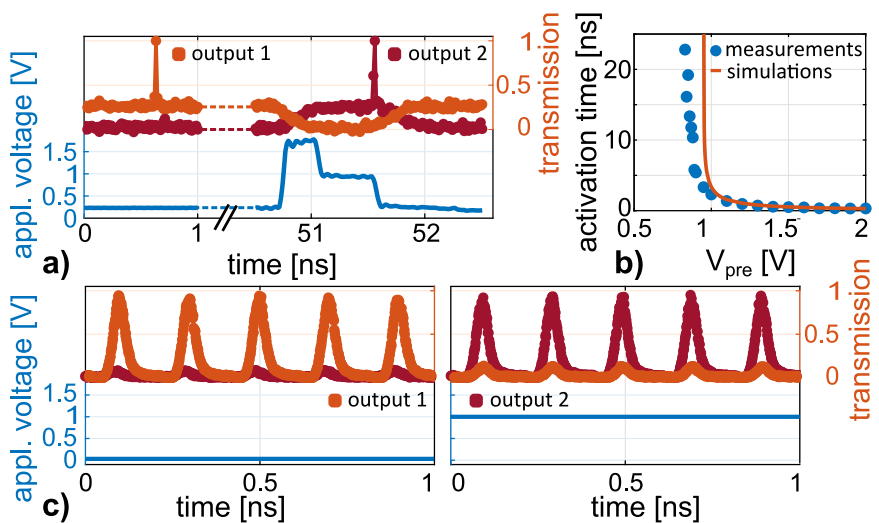

Fig. 8: a) Pulse switching with a 1x2 MZI-switch with $500 \mu \mathrm{m}$ phase-shifter length. Switch activation with a pre-emphasis driving signal. Added $\mathrm{CW}$-light illustrates the transient response during switching operation. b) Measured and simulated time to reach on-state vs. pre-emphasis voltage in MZI-switch. c) Pulse switching with a ring-switch. Transmission at both output ports in the off-state and the on-state $(0.25 \mathrm{~mA}$ diode current).
$0.7 \mathrm{~ns}$ corresponds to a potential switching speed of $1.4 \mathrm{GHz}$, beneficial for the high-speed memory writing targetted in our application.

As discussed before, the ring architecture is an energy-efficient switch alternative to the MZI. Taking into account the optical bandwidth limitations of the ring design (see Section II), we demonstrate pulse-switching with 5 ps-long laser pulses. To avoid attenuation due to free-carrier absorption, the switch is operated on-resonance. The ps pulsed laser available for this experiment has a repetition rate of $5 \mathrm{GHz}$. As the activation time for switching is longer than the pulse period, in this experiment no individual pulses were selected from the pulse train. Thus, the switch is driven in DC operation with a diode current of $0.25 \mathrm{~mA}$. Fig. 8.c shows the measurement results of the switch in off- and on-state respectively indicating the pulse to be primarily present at the targetted output ports.

\section{DISCUSSION}

In this Section, we summarize the performance of switches using carrier-injection-based phase shifters and compare them with the other technologies that utilize the plasma-dispersion effect, namely carrier-depletion and carrier-accumulation. We also discuss potential improvements to further reduce energy consumption.

In order to compare the phase shifter technologies, we choose a balanced MZI-switch, with a $\pi$-phase shift requirement, as a reference. The relevant switch metrics to compare the technologies are benchmarked in Table I covering phase shifter length, loss characteristics for the switch in both off-state (no voltage applied) and on-state (voltage applied) and the switching speed. Furthermore, the dynamic energy required to activate the switch and the static power consumption in onstate are listed in the table. For the carrier-injection technology these values are collected from the presented theoretical and experimental characterization of this work. For calculating the activation energy, we assumed a pre-emphasis driving scheme with a voltage limit of $2 \mathrm{~V}$. As mentioned above, the displayed static power consumption becomes nonrelevant when switching short pulses. The other two technologies were evaluated at the same maximum driving voltage of $2 \mathrm{~V}$. At this voltage we evaluate diode length and dynamic energy consumption $\left(0.5 C \cdot U^{2}\right)$ via the $V_{\pi} \cdot L$ magnitude and the capacitance given in [28], [29]. The insertion loss is related to the provided absorption coefficient and the calculated device length. The static power consumption for these technologies is set to zero, assuming a negligible leakage current under reverse bias. The comparison of all switch metrics in Table I indicates that carrier-injection gives the overall best performance, when operated at a switching speed in the low $\mathrm{GHz}$ range. Thus, this technology is most suitable for pulse switching due to its high efficiency and low insertion loss metrics without sacrificing performance in terms of energy consumption. The comparable dynamic energy consumption of the three platforms is given by the material dependent amount of charge required for a $\pi$-phase shift when using the plasma-dispersion effect. As discussed before, increasing the length of the carrier-injection-based phase shifter further 
TABLE I: Benchmark comparison of performance metrics for photonic switches based on silicon photonics technology. For all technologies the driving voltage was limited to $2 \mathrm{~V}$. Off-state relates to an unbiased device. On-state relates to a phase shifter operated at the driving voltage. Reduced insertion loss is indicated for carrier-accumulation (off-state) and carrier-depletion (on-state) originating from lower free-carrier concentration (not further characterized in [28], [29]).

\begin{tabular}{l|c|c|c|c|c|c}
$\begin{array}{l}\text { Plasma-dispersion } \\
\text { technology }\end{array}$ & $\begin{array}{c}\text { phase shifter } \\
\text { length } \\
{[\mathrm{mm}]}\end{array}$ & $\begin{array}{c}\text { insertion loss } \\
\text { (off-state) } \\
{[\mathrm{dB}]}\end{array}$ & $\begin{array}{c}\text { insertion loss } \\
(\text { on-state) } \\
{[\mathrm{dB}]}\end{array}$ & $\begin{array}{c}\text { dynamic } \\
\text { energy cons. } \\
{[\mathrm{pJ} / \pi]}\end{array}$ & $\begin{array}{c}\text { static } \\
\text { power cons. } \\
{[\mathrm{mW}]}\end{array}$ & $\begin{array}{c}\text { switching } \\
\text { speed } \\
{[\mathrm{GHz}]}\end{array}$ \\
\hline \hline Carrier-injection [this work] & 0.5 & 0.3 & 1.4 & 5.7 & $(0.45)$ \\
Carrier-accumulation [28] & 0.7 & $\begin{array}{c}<5.6 \\
16.0\end{array}$ & $\begin{array}{c}5.6 \\
<16.0\end{array}$ & 5.6 & - \\
Carrier-depletion [28], [29] & 8.0 & $\begin{array}{c}16.4 \\
\text { 1 intrinsic phase shifter cut-off frequency }\end{array}$ & -
\end{tabular}

reduces the energy consumption towards $4 \mathrm{pJ}$ per switching operation and thus below the value presented in Table I.

Further reduction of the energy consumption, as discussed before, is achieved with an unbalanced MZI-switch. The static phase imbalance between the Mach-Zehnder arms results in improved performance in terms of phase shift requirements. This becomes clear when plotting the extinction ratio of an unbalanced switch (at output 2; see Section III) as a function of energy consumption as shown in Fig. 9. Here, extinction ratio is defined as the ratio of switch transmission in on-state and off-state (same port), while the energy consumption is evaluated using Eq. 7. As indicated by the figure, the reduced phase shift requirements for switching $(0.4 \pi)$ results in a reduction in energy consumption of around $35 \%$. Even smaller energy consumption may be achieved with a switch that is operated with push-pull drive [24].

Ring-based switch designs have the advantage of smaller phase shift requirements to achieve switching. In general, these devices benefit from operation close to sharp resonances that allows a large variation in transmission with only small resonance detuning. The sharper the resonance of a ring (higher Q-factor), the smaller the required detuning to reach a desired extinction ratio (ER). This has been evaluated for three ring-switches with different Q-factors, that are modified by changing the coupling gap between ring and bus waveguide [25]. With a change of the gap from 190 to $290 \mathrm{~nm}$, the Q-factor increases from 1600 to 6400 . The relevant parameters of the ring-switches are given in Table II together with the corresponding optical bandwidths of the switch. The extinction ratio of the devices is extracted from

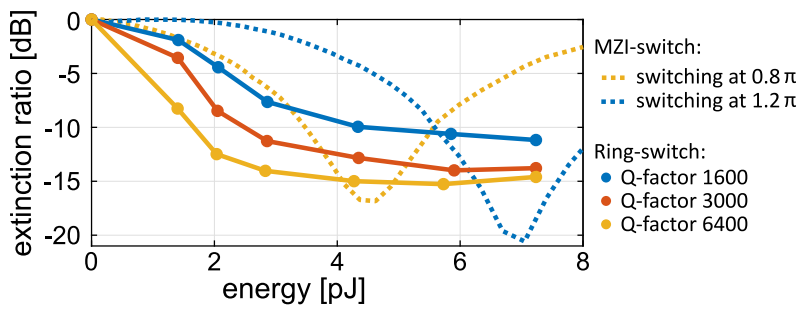

Fig. 9: Extinction ratio vs. energy consumption of unbalanced MZI-switches and ring-switches. The Q-factor of the ringswitch is controlled by changing the coupling gap between bus waveguide and ring-resonator $(15 \mu \mathrm{m}$ radius $)$. pulse switching experiments as described in Section II and the results are shown in Fig. 9. The benefits of a larger Q-factor are clearly visible in the figure. First, it offers more energy efficient devices for a given extinction ratio and second, it leads to an overall increased extinction ratio which relates to the reduced optical bandwidth of the device.

In the literature ring-based modulators have been shown with a much lower energy consumption than presented here. The consumed energy of these modulators is typically expressed in terms of energy per bit with the bit information encoded in an amplitude modulation scheme (e.g. on-off keying). For ring modulators the reduction in energy consumption commonly relies on the combination of high Q-factor and reduced ER. For example, a ring-modulator $(Q \sim 25000)$ has been reported with an energy consumption of $37.1 \mathrm{fJ} / \mathrm{bit}$ [30], while even lower values (3 fJ/bit) are achieved with a high-Q disk-modulator benefiting from a small ER of only $3.2 \mathrm{~dB}$ [31]. Similar energy consumption will be achieved with the presented ring-switches when using reduced ER and higher Q-factors, as suggested by Fig. 9. However, the modulators as presented in the literature do not fulfill our requirements. When switching short pulses with ring-switches, the increased energy efficiency related to higher Q-factors is opposed with a larger insertion loss. This is caused by the bandpass-like filter properties of the ring system, that becomes relevant for a combination of short pulses (wide signal bandwidth) and high-Q rings (narrow filter bandwidth). For example, we evaluate the insertion loss (on-resonance) of a switched $5 \mathrm{ps}$ pulse (signal bandwidth: $88 \mathrm{GHz}, 0.7 \mathrm{~nm}$ ) with the aforementioned rings (Q-factors between 1600 and 6400). Table II shows the increased insertion-loss of $1.4 \mathrm{~dB}$ for the largest Q-factor. Given the presented results, we

TABLE II: Relation of Q-factor and optical bandwidth in dependence of the coupling gap between bus waveguide and ring-resonator $(15 \mu \mathrm{m}$ radius). The insertion loss is measured for 5 ps pulses on-resonance (signal bandwidth: $88 \mathrm{GHz}$, $0.7 \mathrm{~nm})$.

\begin{tabular}{l|c|c|c} 
Coupling gap [nm] & 190 & 240 & 290 \\
\hline \hline & 1600 & 3000 & 6400 \\
Q-factor & 0.99 & 0.51 & 0.24 \\
Optical bandwidth [nm] & 124 & 64 & 30 \\
Optical bandwidth [GHz] & $\sim 0$ & 0.3 & 1.4 \\
Insertion loss (5 ps pulse) [dB] &
\end{tabular}


conclude that the ring-switch with a gap of $240 \mathrm{~nm}$ gives the best trade-off between energy efficiency and insertion loss.

\section{SUMmARY AND CONCLUSION}

In this work, we presented silicon photonic switches activated by carrier injection. The devices are characterized and methodically optimized for the routing of picosecond pulses with maximum energy efficiency. We have used a small-signal analysis to measure the voltage-dependent capacitance, which is essential to accurately assess the energy consumption of these devices. Additionally, we have correlated the energy consumption to the amount of moved carriers and optical phase shift. We have demonstrated a novel way to classify energy dissipation in all electronic components of the switch (diode, resistance, capacitance) by using a large-signal model. This experimentally verified model allows to optimize the driving scheme for a sixfold better energy efficiency and to identify the negligible impact of static power consumption during pulse switching. We have compared phase shifters of 250 to $1400 \mu \mathrm{m}$ length and have found that longer devices benefit from lower carrier concentrations. This leads to a $50 \%$ more efficient MZI-switch with an energy consumption down to $4 \mathrm{pJ}$ per switch operation using optimized driving schemes. In addition, we found ring-switches allow to further reduce the energy consumption by $50 \%$ compared to the optimized MZI-switches and discussed how the Q-factor impacts the trade-off between energy efficiency and optical bandwidth. We have highlighted the sub-nanosecond switching speed and low insertion loss of carrier-injection-based switches (off-state $0.3 \mathrm{~dB}$, on-state $1.4 \mathrm{~dB}$ ) and compared this technology to similar platforms based on the plasma-dispersion effect in silicon. We have compared the potential of these platforms for realizing photonic routing networks that provide low insertion loss, high energy efficiency and allow to route short optical pulses (100 fs-10 ps) with a repetition rate in the order of $1 \mathrm{GHz}$. We found that the carrier-injection-based switching platform shows the overall best performance and we recommend this technology as the way to go for pulse routing in applications like all-optical memory writing.

\section{APPENDIX A}

The energy consumption of a carrier-injection-based phase shifter can be highly reduced by using an electrical driving scheme with pre-emphasis. With an optimized driving scheme the energy efficiency of the device can be increases by a factor larger than six as displayed in Fig. 10 for a $\pi$-phase shift target.

\section{REFERENCES}

[1] M. L. M. Lalieu, M. J.G. Peeters, S. R.R. Haenen, R. Lavrijsen, and B. Koopmans, "Deterministic all-optical switching of synthetic ferrimagnets using single femtosecond laser pulses," Phys. Rev. B, vol. 96, no. 22, pp. $1-5,2017$

[2] K. Vahaplar, A. M. Kalashnikova, A. V. Kimel, S. Gerlach, D. Hinzke, U. Nowak, R. Chantrell, A. Tsukamoto, A. Itoh, A. Kirilyuk, and T. Rasing, "All-optical magnetization reversal by circularly polarized laser pulses: Experiment and multiscale modeling," Phys. Rev. B, vol. 85, no. 10, pp 104402, 2012

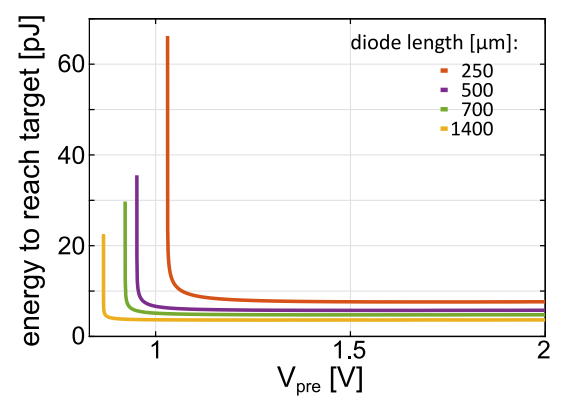

Fig. 10: Calculation of the energy consumption based on largesignal modelling. $V_{\text {pre }}$ indicates the pre-emphasis voltage level of the pre-emphasis-based driving scheme.

[3] J. Y. Chen, L. He, J. P. Wang, and M. Li, "All-Optical Switching of Magnetic Tunnel Junctions with Single Subpicosecond Laser Pulses," Phys. Rev. Appl., vol. 7, no. 2, pp. 2-7, 2017

[4] F. Y. Gardes, G. T. Reed, N. G. Emerson, and C. E. Png, "A sub-micron depletion-type photonic modulator in Silicon On Insulator," Opt. Express, vol. 13 , no. 22 , pp. $8845-8854,2005$

[5] A. Liu, L. Liao, D. Rubin, H. Nguyen, B. Ciftcioglu, Y. Chetrit, N. Izhaky, and M. Paniccia, "High-speed optical modulation based on carrier depletion in a silicon waveguide," Opt. Express, vol. 15, no. 2, pp. 660668,2007

[6] A. Liu, R. Jones, L. Liao, D. Samara-Rubio, D. Rubin, O. Cohen, R. Nicolaescu, and M. Paniccia., "A high- speed silicon optical modulator based on a metal-oxide-semiconductor capacitor," Nature, vol. 427, pp. 615-618, 2004

[7] Q. Xu, B. Schmidt, S. Pradhan, and M. Lipson, "Micrometer-scale silicon electro-optic modulator," Nature, vol. 435, pp. 325-327, 2005

[8] J. Van Campenhout, W. M. J. Green, S. Assefa, and Y. A. Vlasov, "Low-power, 2x2 silicon electro-optic switch with 110-nm bandwidth for broadband reconfigurable optical networks," Opt. Express, vol. 17, no. 26, pp. 24020-24029, 2009

[9] C.J. Krückel, H. Becker, Y. Ban, M. J. R. Heck, J. Van Campenhout, and D. Van Thourhout, "Towards High-Speed Energy-Efficient Pulse-Switching Networks Implemented in Carrier-Injection-Based SiPhotonics," Conference on Lasers and Electro-Optics (CLEO) Europe, Munich, Germany, June 2019

[10] C.J. Krückel, H. Becker, M. J. R. Heck, J. Van Campenhout, and D. Van Thourhout, "Design optimization for energy-efficient pulse-switching networks in carrier-injection based Si-photonics," European Conference on Optical Communications (ECOC), Dublin, Ireland, September 2019

[11] D. Patel, S. Ghosh, M. Chagnon, A. Samani, V. Veerasubramanian, M. Osman, and D. V. Plant, "Design, analysis, and transmission system performance of a $41 \mathrm{GHz}$ silicon photonic modulator," Opt. Express, vol. 23, no. 11, pp. 14263-14287, 2015

[12] W. M. J. Green, M. J. Rooks, L. Sekaric, and Y. A. Vlasov, "Ultracompact, low RF power, $10 \mathrm{~Gb} / \mathrm{s}$ silicon Mach-Zehnder modulator," Opt. Express, vol. 15, no. 25, pp. 17106-17113, 2007

[13] X. Chen, Y.-S. Chen, Y. Zhao, W. Jiang, and R. T. Chen, "Capacitorembedded $0.54 \mathrm{pJ} / \mathrm{bit}$ silicon-slot photonic crystal waveguide modulator," Opt. Letters, vol. 34, no. 5, pp. 602-604, 2009

[14] P. Dong, S. Liao, D. Feng, H. Liang, D. Zheng, and M. Asghari, "Low Vpp, ultralow-energy, compact, high-speed silicon electro-optic modulator," Opt. Express, vol. 17, no. 25, pp. 22484-22490, 2009

[15] D. Miller, "Energy consumption in optical modulators for interconnects," Opt. Express, vol. 20, no. S2, pp. A293-A308, 2012

[16] S.Manipatruni, Q.Xu, and M.Lipson, "PINIP based high-speed highextinction ratio micron-size silicon electrooptic modulator," Opt. Express, vol. 15, no. 20, pp. 13035-13042, 2007

[17] Q.Xu, S.Manipatruni, B.Schmidt, J.Shakya, and M.Lipson, "12.5 Gbit/s carrier-injection-based silicon micro-ring silicon modulators," Opt. Express, vol. 15 , no. 2 , pp. 430-436, 2007

[18] R. A. Soref, and B. R. Bennet, "Electrooptical Effects in Silicon," IEEE J. Quantum Electron., vol. QE-23, no. 1, pp. 123-129, 1987

[19] M. Nedelijkovic, R. Soref and G. Z. Mashanovich, "Free-Carrier Electrorefraction and Electroabsorption Modulation Predictions for Silicon Over the 1-14- $\mu m$ Infrared Wavelength Range," IEEE Photonics J., vol. 3, no. 6, pp. 1171-1180, 2011 
[20] "Si-Photonics iSiPP50G" [Online]. Available: https://europracticeic.com/mpw-prototyping/siphotonics/imec/

[21] G. R. Zhou, M. W. Geis, S. J. Spector,F. Gan, M. E. Grein, R. T Schulein, J. S. Orcutt, J. U. Yoon, D. M. Lennon, T. M. Lyszczarz, E. P. Ippen, and F. Kaertner, "Effect of carrier lifetime on forward-biased silicon Mach-Zehnder modulators," Opt. Express, vol. 16, no. 8, pp. 52185226, 2008

[22] R. F. Pierret, "Semiconductor Device Fundamentals," Addison Wesley, 1996

[23] R. S. Tucker, and K. Hinton, "Energy consumption and energy density in optical and electronic signal processing," IEEE Photonics J., vol. 3, no. 5 , pp. 821-833, 2011

[24] N. Dupuis, A. V. Rylyakov, C. L.Schow, D. M. Kuchta, C. W. Baks, J. S. Orcutt, D. M Gill, W. M. J. Green, and B. G. Lee, "NanosecondScale Mach - Zehnder-Based CMOS Photonic Switch Fabrics," J. Light. Technol., vol. 35, no. 4, pp. 615-623, 2017

[25] W. Bogaerts, P. De Heyn, T. Van Vaerenbergh, K. De Vos, S. K. Selvaraja, T. Claes, P. Dumon, D. Van Thourhout, and R. Baets, "Silicon microring resonators," Laser Photonics Rev., vol. 6, no. 1, pp. 47-73, 2012

[26] S.Tanaka, T.Usuki, and Y.Tanaka, "Accurate SPICE Model of ForwardBiased Silicon PIN Mach-Zehnder Modulator for an Energy-Efficient Multilevel Transmitter," J. Light. Technol., vol. 36, no. 10, pp. 1959-1959, 2018

[27] "Simulink." [Online]. Available: https://nl.mathworks.com/products/simulink.html

[28] S. S. Azadeh, F. Merget, S. Romero-García, A. Moscoso-Mártir, N. von den Driesch, J. Müller, S. Mantl, D. Buca, and J. Witzens, "Low $\mathrm{V}_{\pi}$ Silicon photonics modulators with highly linear epitaxially grown phase shifters," Opt. Express, vol. 23, no. 18, pp. 23526-23550, 2015

[29] S. S. Azadeh, J. Müller, F. Merget, S. Romero-Garcia, B. Shen, and J. Witzens, "Advances in Silicon photonics segmented electrode MachZehnder modulators and peaking enhanced resonant devices," Proc. SPIE9288, 928817, 2015

[30] S. Manipatruni, K. Preston, L. Chen, and M. Lipson, "Ultra-low voltage, ultra-small mode volume silicon microring modulator," Opt. Express, vol. 18 , no. 17 , pp. $18235-18242,2010$

[31] M. R. Watts, W. A. Zortman, D. C. Trotter, R. W. Young, and A. L. Lentine, "Vertical junction silicon microdisk modulators and switches," Opt. Express, vol. 30, no. 22, pp. 33-38, 2011

Clemens J. Krückel received his M.Sc. degree in electrical engineering from the Technical University Hamburg-Harburg in 2012 and his Ph.D. degree in 2017 from Chalmers University of Technology for his work on nonlinear optics in silicon nitride waveguides. He is currently a Postdoctoral Researcher with Ghent University-IMEC where he is working towards reconfigurable optical networks in SOI technology. His work is part of the European SPICE project that explores all-optical switching of magnetic memory cells. His research interests include nanofabrication technologies, carrier-dynamics in semiconductor devices and integrated optoelectronics.
Yoojin Ban received the B.Sc. and M.Sc. degrees in electrical and electronic engineering from Yonsei University, Seoul, South Korea, in 2013 and 2015, respectively. Her M.Sc. thesis topic was on silicon micro-ring modulator modeling. Currently she is working in IMEC, Belgium as a R\&D engineer specialized in high speed Si based modulators on silicon photonics since 2015. In parallel, she is currently Doctoral Researcher in IDLab, Ghent University, Ghent, Belgium. Her research topic is about integrated optical transmitter design using CMOS and Silicon Photonics Technology.

Martijn J. R. Heck received the M.Sc. degree in applied physics and the Ph.D. degree in electrical engineering from the Eindhoven University of Technology, the Netherlands, in 2002 and 2008, respectively. From 2007 to 2008, he was a Postdoctoral Researcher at the COBRA Research Institute in Eindhoven, where he was engaged in the development of a technology platform for active-passive integration of photonic integrated circuits. From 2008 to 2009, he was with the Laser Centre, Vrije Universiteit in Amsterdam, the Netherlands, where he was involved in the development of integrated frequency-combs generators. From 2009 to 2013, he was Postdoctoral Researcher and Associate Director of the Silicon Photonics Center at the University of California Santa Barbara, USA, where he was involved in photonic integrated circuits based on the heterogeneous integration of silicon, silica and III/V photonics.

\section{Joris Van Campenhout}

Dries Van Thourhout received the degree in physical engineering and the Ph.D. degree from Ghent University, Ghent, Belgium in 1995 and 2000 respectively. From Oct. 2000 to Sep. 2002 he was with Lucent Technologies, Bell Laboratories, New Jersey, USA, working on the design, processing and characterization of $\mathrm{InP} / \mathrm{InGaAsP}$ monolithically integrated devices. In Oct. 2002 he joined the Department of Information Technology (INTEC), Ghent University, Belgium. Currently he is member of the permanent staff of the photonics group. Since 2008 he has a position as full time professor. He is chair of the joint UGent-VUB Master in Photonics program and lecturer or co-lecturer for four courses within the Ghent University (Microphotonics, Advanced Photonics Laboratory, Photonic Semiconductor Components and Technology). He is coordinating the cleanroom activities of the research group and coordinating of the NAMIFAB centre of expertise. His research focuses on the design, fabrication and characterization of integrated photonic devices. Main topics involve Silicon nanophotonic devices and the integration of novel materials (III-V, graphene, ferro-electrics, quantum dots, ...) on these waveguides to expand their functionality. He is working on applications for telecom, diatom, optical interconnect and sensing.
Hanna Becker studied physics at the University of Konstanz and received the B.Sc. and M.Sc. degree in 2012 and 2015, respectively. She spent three month at the Marine Biological Laboratory (MBL) in Woods Hole, US, for her Bachelor project and half a year in the electronics $R \& D$ of BaslerAG working on quality assurance and testing of cameras as an intern during her master studies. She returned to the University of Konstanz to work on a femtosecond-fiber laser system for her master thesis. She received her PhD degree in June 2020 from Aarhus University for her work on silicon photonic grating couplers and contributions to the SPICE project, integrating silicon photonics with optical MRAM technology. She currently continues her research towards integrated MRAM technology as a Postdoctoral Researcher at Aarhus University and additionally is part of the InPulse project, establishing a manufacturing pilot line for Indium-Phosphide photonic integrated circuits. 